\title{
Directed motor actions and choice signalling drive cortical acetylcholine dynamics.
}

Authors: Jing Zou ${ }^{1}$, Simon Trinh ${ }^{1}$, Andrew Erskine ${ }^{1}$, Miao Jing ${ }^{2}$, Jennifer Yao ${ }^{1}$, Stefanie Walker ${ }^{1}$, Yulong $\mathrm{Li}^{3-5}$, Samuel Andrew Hires ${ }^{1 *}$

\section{Affiliations:}

${ }^{1}$ Department of Biological Sciences, Section of Neurobiology, University of Southern California, Los Angeles, California, United States of America.

${ }^{2}$ Chinese Institute for Brain Research, Beijing, China.

${ }^{3}$ State Key Laboratory of Membrane Biology, Peking University School of Life Sciences, Beijing, China.

${ }^{4} \mathrm{PKU}-$ IDG/McGovern Institute for Brain Research, Beijing, China.

${ }^{5}$ Peking-Tsinghua Center for Life Sciences, Academy for Advanced Interdisciplinary Studies, Peking University, Beijing, China

* Correspondence to: shires@usc.edu

\begin{abstract}
Numerous cognitive functions including attention, learning, and plasticity are influenced by the dynamic patterns of acetylcholine release across the brain. How acetylcholine mediates these functions in cortex remains unclear, as the spatiotemporal relationship between cortical acetylcholine and behavioral events has not been precisely measured across task learning. To dissect this relationship, we quantified motor behavior and sub-second acetylcholine dynamics in primary somatosensory cortex during acquisition and performance of a tactile-guided object localization task. We found that acetylcholine dynamics were spatially homogenous and directly attributable to whisker motion and licking, rather than sensory cues or reward delivery. As task performance improved across training, acetylcholine release to the first lick in a trial became dramatically and specifically potentiated, paralleling the emergence of a choice-signalling basis for this motor action. These results show that acetylcholine dynamics in sensory cortex are driven by directed motor actions to gather information and act upon it.
\end{abstract}

\section{Introduction}

Acetylcholine is a major neuromodulator in the brain that influences diverse cognitive functions that span timescales, including selective attention $\underline{1}, \underline{2}, \underline{3}, \underline{4}$, arousal $\underline{5}, \underline{6}, \underline{7}, \underline{\mathbf{8}}$, reinforcement learning $\underline{5}$, $9 ; 10,11,12$ and neural plasticity $13,14,15,16$. Many of these functions are mediated through acetylcholine's influence on cortical neurons and circuits $\underline{17}, \underline{18}$. Cholinergic projections to cortex arise from multiple basal forebrain (BF) nuclei that contain neuronal subgroups with distinct projection specificity and arbor distributions within and across projection areas $\underline{\mathbf{7}}, \underline{\mathbf{1}}, \underline{\mathbf{2 0}}$. Individual nuclei also show significant differences in the behavioral events which correlate with their activity patterns $\underline{7}, \underline{21}$, suggesting a topography of functions. Observation of the spatiotemporal 
dynamics of acetylcholine in cortex would further our understanding of how these convergent cholinergic inputs influence cortically regulated cognitive functions.

The release of acetylcholine on cortical targets is classically considered to regulate arousal and attention $17, \underline{18}$, in part through action on cortex. Increased cortical acetylcholine levels are associated with $\underline{22}$ and required for induction of cortical desynchronization during active sensing $\underline{\mathbf{2 3}}$. Acetylcholine release causes layer-specific modulation of responses in primary somatosensory cortex (S1) to whisker stimulation ${ }^{1} \underline{24}$, enhances sensory evoked responses in $\mathrm{A} 1 \underline{\underline{3}}$ and $\mathrm{V} 1 \underline{\mathbf{2 5}}, \underline{\mathbf{2 6}}$, and suppresses spontaneous activity in $\mathrm{S} 1$ during whisker movement $\underline{23}$, which improves stimulus discriminability and provides a potential mechanism for selective attention.

The role of acetylcholine in reinforcement learning is becoming better appreciated through accumulating studies identifying which cholinergic neurons fire when during task acquisition and execution. Cholinergic neurons in BF respond ${ }^{11}$ and cortical acetylcholine transients are evoked to reinforcement-predictive cues $\underline{\mathbf{5}}$. However, the extent to which association learning sculpts the response to reinforcement-predictive cues varies across reports, with such stimuli driving increasing amounts of cholinergic activity in BF (tones and punishment $\frac{16}{16}$, odors and reward $\frac{12}{2}$ ) and in nucleus basalis (NB) to basolateral amygdala (BLA) projections (tones and reward ${ }^{11}$ ) across training, contrasting with the finding that reward-predictive tones show stable acetylcholine release in BF with learning 21 . Functionally, persistent activity of cholinergic neurons bridges delays between conditioned stimuli and reinforcement signals, allowing cortical acetylcholine-dependent frequency-specific potentiation and map plasticity in A1 1 . . Moreover, BF cholinergic input is necessary ${ }^{9}$ and sufficient ${ }^{10}$ for cue-evoked reward timing signals to develop in V1 across associative learning. As for reinforcers, cholinergic neurons throughout BF strongly respond to negative reinforcement $12,16,21,27,28$, but are inconsistently reported to respond to positive reinforcers like reward. In primates, $70 \%$ of all BF neurons were significantly modulated in a choice period, but only $25 \%$ in a reward period $\underline{29}$. In rats, reward-induced acetylcholine release was not detected in $\mathrm{MPFC}{ }^{5}$. However, recent advances in cell-type specific recording techniques have revealed that cholinergic neurons within BF do respond to positive reinforcers, scaled by reinforcement surprise ${ }^{27}$ and encode valence-free reinforcement error $\underline{12}$.

Motor actions are also associated with cholinergic activity, which could be challenging to disentangle from cue and reward-driven effects due to sensory-motor feedback loops. Cortical

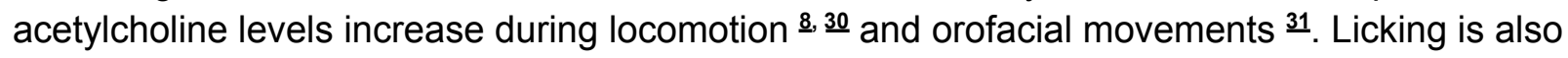
reported to drive cholinergic activity, though reports vary from strong acetylcholine release at lick bout onset $\underline{28}$ and offset $\underline{21}$, to weak release to licks $\underline{12}$ to none at all $\underline{27}$. This variability may arise from experimental differences in task conditions and sensory modalities or may reflect a compartmentalization of acetylcholine functions based on nucleus, cell-type, and projection targets. Regardless, since licking patterns are strongly influenced by reward expectation and delivery ${ }^{32}$, dissociating sensory cues and reward from these motor actions is crucial for interpreting acetylcholine's role in reinforcement learning. 
Here we sought to constrain how acetylcholine influences sensory cortex by measuring spatiotemporal dynamics of acetylcholine in S1, an area that undergoes remarkable representational plasticity during learning ${ }^{33}$. We directly image sub-second changes in acetylcholine concentration using a GPCR Activation Based sensor (GRAB-ACh3.0; 30) broadly expressed on the surface of cortical neurons, bypassing the organizational complexity of cholinergic afferents. High-speed videography during active whisker-guided object location discrimination allows dissociation of cue and stimulus from tightly coupled motor responses, while our response design allows dissociation of choice signalling and reward delivery from the motor action of licking. Recording across weeks of training, we identify the behavioral correlates of acetylcholine release in S1 during task performance and how tactile discrimination learning specifically reorganizes those dynamics in the transition from novice to master.

\section{Results}

To measure the dynamics of acetylcholine in primary somatosensory cortex during tactile discrimination learning, we employed a go/no-go single-whisker object localization task (Figure 1A ${ }^{34}$ ). We trained water-restricted head-fixed mice ( $n=8$ mice) to search with a whisker for the position of a thin steel pole presented in one of two locations along an anteroposterior axis during a one second sampling period (Figure 1B), and to lick for a water reward during a subsequent one second answer period if the pole was in the posterior location. Mice were cued to the presentation and removal of the pole by the sound of a pneumatic valve. Licking during the sampling period had no consequences, while licking in the answer period determined trial outcome and extended the duration of the pole presentation.

Mice were trimmed to their $\mathrm{C} 2$ whisker at least one week prior to onset of imaging experiments. All mice reached task mastery (>70\% performance for 3 consecutive sessions) within 1-3 weeks of training (mean $10.75 \pm 2.02$ sessions; mean $3373.75 \pm 754.09$ (standard error) trials Figure 1C). Trimming of the C2 whisker after task mastery dropped performance to chance (Figure 1D), demonstrating the whisker-dependence of the task. Expert mice initiated whisker exploration upon the pole presentation and withdrawal cue sounds (Figure 1E, F, SFigure 1A). Since whisking amplitude was physically restricted when the pole was in the go-associated proximal position, we examined the whisking patterns of go trials vs. no-go trials separately. For both positions, trials with licks during the sampling and answer periods (i.e. Hit and False Alarm) had more sustained whisking than trials without licks (i.e. Miss and Correct Rejection; Figure 1G). Lick rates on Hit and False Alarm trials were indistinguishable during the sampling period and diverged during the answer period once water was dispensed due to reward collection on the Hit trials (Figure 1H). Licking rates on Correct Rejection and Miss trials were zero by construction during the answer period, with occasional licks outside this period.

Acetylcholine dynamics were measured using two-photon imaging of superficial layers of S1. The C2 whisker barrel was targeted for viral injection of AAV9-GRAB-ACh3.0 via intrinsic signal imaging (SFigure 2A). Behavioral training commenced three weeks post injection, when strong fluorescence signals were present in all mice (Figure 2A). To minimize the impact of a rapid partial dimming of fluorescence following illumination onset (SFigure 2B), two-photon imaging and illumination (15.44 fps, $940 \mathrm{~nm}, 30-60 \mathrm{~mW}$ out of objective) was continuous from the 
session start, and the first 100 seconds of the session were excluded from analysis. Phasic increases in fluorescence across the entire field of view were observed shortly after most, but not all onsets of pole presentation (Figure 2B). These fluorescence dynamics diverged across trial outcomes (Figure 2C). Hit and False Alarm trials showed a strong, similar increase in signal during the sampling and answer periods (example session, SFigure 2C; grand mean of expert sessions Figure 2D). This was followed by a secondary response that, on average, persisted into the inter-trial period. In contrast, Miss and Correct Rejection trials on average showed two short latency, short duration increases in fluorescence following pole presentation and withdrawal. Trial type averages within the primary whisker column and in the surround were identical for all trial types (Figure 2E) supporting mesoscale spatial homogeneity in acetylcholine dynamics within S1.

To investigate potential triggers of acetylcholine release in cortex, we compared the acetylcholine dynamics across trial types to several classes of behavioral events, including pole presentation and withdrawal cues, whisker exploration, licking, and reward. All trial types shared a common time for pole presentation, and all trial types showed a small, short latency (2-3 frames, 130-195ms) increase in acetylcholine aligned to that cue (Figure 3A). Lick trials showed much bigger and longer transients immediately following this initial hump. Trials without licks in the answer period had a common pole withdrawal time, while trials with answer licks had a variable withdrawal time. Alignment to pole withdrawal again showed a sharp upward transient of acetylcholine across all trial types, though this was overlaid on longer duration acetylcholine dynamics (Figure 3B).

Was acetylcholine release driven by the pole presentation cue per se, or was it driven by a motor response to the cue (Figure 1E,F)? To test this, we examined the covariation of cue-evoked whisking with cue-associated acetylcholine dynamics. We restricted our analysis to no-lick trials to avoid potential confounds of licking-driven acetylcholine responses. We sorted acetylcholine responses in no-lick trials by the average amplitude of the whisker motion within 500 milliseconds after the pole presentation cue (Figure 3C, D). A fraction of trials (23.9\% mean $\pm 21.8 \%$ per mouse) did not evoke whisker motion ( $<2^{\circ}$ mean amplitude) upon pole presentation, with most producing a range of whisking vigor (SFigure 3A). In trials without cue-evoked whisking, there was no acetylcholine release following the cue $(0.01 \%$ mean $\Delta \mathrm{F} / \mathrm{F}$ for whisking amplitude $<2^{\circ}$ ), while trials with cue-evoked whisking showed a positive relationship between whisking amplitude and acetylcholine response $(0.58 \%$ mean $\Delta \mathrm{F} / \mathrm{F}$ for whisking amplitude $>5^{\circ} ; 0.08 \%$ mean increase in $\Delta F / F$ per degree of amplitude (Figure 3E, SFigure 3B). These findings were recapitulated in pole withdrawal-cued whisking and acetylcholine responses (SFigure 3C-E). This implies that whisking, rather than whisker-pole contact drove the responses, since whisking after pole withdrawal rarely causes pole touches. These data demonstrate that cue-associated acetylcholine release in S1 is directly proportional to the magnitude of the motor response (i.e. exploratory whisking) evoked by that cue. We conclude that the motor response to cue, rather than the cue itself, drives cue-associated acetylcholine transients in S1. 
The striking difference in acetylcholine dynamics across trial types (Figure 2C-E), in particular Hit and False Alarm versus Miss and Correct Rejection, suggested that licking could be a powerful driver of acetylcholine release in S1. We aligned trials across expert sessions to the time of first lick, regardless of trial type or if the lick occured in the answer period, and sorted by the number of licks in the trial (Figure 4A,B). Trials with no licks were aligned to the time of the median first lick. On trials with licks, there was a sharp increase in acetylcholine level beginning shortly before lickport detection of the first lick, which lasted 1-2 seconds (Figure 4C). This lick response dwarfed the cue-associated transients on the no-lick trials (Figures 2E, 4C). Licking preceded rhythmically at a regular modal inter-lick interval of $155 \mathrm{~ms}$ in expert mice (Figure 4D). The first lick drove a profound increase in mean acetylcholine over the following second, jumping from $0.35 \% \pm 0.26 \%$ on no-lick trials to $1.60 \% \pm 0.42 \%$ with a single lick (Figure 4E), an increase of $1.25 \% \Delta \mathrm{F} / \mathrm{F}$ over baseline. Subsequent licks caused smaller increases in mean acetylcholine over this period at a rate of $0.28 \% \Delta \mathrm{F} / \mathrm{F}$ per additional lick. Similarly, the duration of the lick-induced transient was $1.46 \pm 0.65 \mathrm{~s}$ for a single lick.

Subsequent licks extended this transient $116 \mathrm{~ms}$ per lick (Figure 4F), somewhat less than the inter-lick interval (Figure 4D). This demonstrates that the primary driver of acetylcholine release in $\mathrm{S} 1$ was the onset of a licking bout, with the vigor of licking modulating the magnitude and duration of the release.

The early lick-evoked acetylcholine transients were followed by a longer late acetylcholine rebound that began 1.5-3 seconds after the first lick (Figure 2C-E). We hypothesized that the late response was driven by reward delivery, as observed in BF cholinergic neurons ${ }^{12}, 27$ and BF to BLA projections ${ }^{11}$. To test this, we compared the first lick aligned responses on Hit trials (which are rewarded) and False Alarm trials (which are unrewarded). Against our hypothesis, there was no significant difference in the amplitude of the late acetylcholine response between Hit and False Alarm trials (Figure 5A), although there was a small, but significant increase on Hit trials during a one second period following the first lick. Could this difference be explained by reward delivery?

Rewards are only distributed upon a correct lick in the answer period. Hit trials had higher acetylcholine levels than False Alarms during the answer period, but also had more licks (Figure 5B,C). To control for increased acetylcholine release caused by additional licking (Figure 4E), we pairwise compared mean acetylcholine levels of Hit and False Alarm trials during the answer period for matched numbers of licks in the period. Hit and False Alarm acetylcholine levels were essentially identical after accounting for the difference in lick count (Figure 5D). We conclude that reward delivery does not cause acetylcholine release in S1,

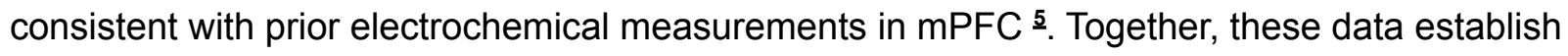
that the main driver of acetylcholine release in S1 during tactile-guided choice behavior is execution of the choice-signalling action, secondary drivers are exploratory whisker motion and additional licking, while task initiation cues and reward delivery have no direct effect.

Acetylcholine regulates attention $\frac{35}{3}$, which may change with task performance, familiarity, or learning. It follows that training might increase acetylcholine release concurrently with improved performance. On the other hand, training might reduce acetylcholine release, as a 
familiar task may require less attentional resources to solve or reduced neural plasticity once established. To address these possibilities, we compared the acetylcholine dynamics for the first three sessions of the full task training and the final three expert sessions in each mouse. We found that the initial lick-triggered acetylcholine release was nearly twice as large in expert sessions compared to early training sessions ( $\Delta \mathrm{F} / \mathrm{F} 3.07 \pm 1.01 \%$ std expert vs. $1.67 \pm 0.65 \%$ std early; Figure 6A). The magnitude of the increase was directly proportional to session performance with mean $\Delta F / F$ increasing $0.53 \%$ per $10 \%$ increase in correct rate (Figure $6 \mathrm{~B}$ ). This increase occurred even though expert mice licked fewer times (Figure 6C,D) and at the same pace (Figure 6E) as compared to early sessions. This increase of acetylcholine signal after training was also not due to an increase of GRAB-ACh sensor expression as seen by the following internal control. On no-lick trials, whisking-associated acetylcholine release slightly increased following the pole-presentation cue, and slightly decreased following the pole-withdrawal cue (Figure 6F). This closely matched the change in whisking to those cues following training (Figure 6G). Thus, the relationship between acetylcholine fluorescence change and whisker motion was stable across training. Finally, we examined whether training induced potentiation of all licks, or only the first lick (i.e. the choice-signalling lick in expert sessions), by repeating the analysis of Figure 4 on early sessions of the same cohort of mice. In contrast to experts (Figure 4E), the first lick in early sessions drove a modest increase in acetylcholine from $0.14 \% \pm 0.3 \%$ on no-lick trials to $0.50 \% \pm 0.52 \%$ with a single lick (Figure $6 \mathrm{H})$, an increase of $0.36 \% \Delta \mathrm{F} / \mathrm{F}$ over baseline. Thus, training induced a nearly $3.5 \mathrm{x}$ increase in acetylcholine response over baseline to the first lick (Figure 6I). Subsequent licks caused a $0.26 \%$ increase in $\Delta F / F$ per additional lick versus $0.28 \% \Delta F / F$ per lick in expert mice (Figure $6 \mathrm{H})$. The nearly identical slope, but shifted offset in the licking to acetylcholine relationship leads us to conclude that training in tactile-guided choice behavior induces a dramatic and selective potentiation of acetylcholine release in S1 to a choice-signalling action and this potentiation is correlated to improved task performance.

\section{Discussion}

Recording and manipulation of cholinergic neurons originating in BF nuclei 26; 27; 36; 37 has established the importance of cholinergic signalling on multiple brain functions. However, the complexity of BF organization $7 ; 19 ; 20$ has posed a challenge in linking specific cognitive functions to acetylcholine dynamics in specific projection targets. By recording acetylcholine dynamics directly in S1 (Figure 2) during whisker-guided object localization (Figure 1), we discovered surprising differences in the triggers and dynamics between previously observed BF nuclei and this cortical target essential for tactile discrimination ${ }^{38}$. Nearly all acetylcholine release in S1 was attributable to directed motor actions (Figures 3, 4) rather than sensory input (Figure 3) or reward delivery (Figure 5). Moreover, as task performance improved across training, acetylcholine release to the first lick in a trial became dramatically and specifically potentiated (Figure 6), paralleling the emergence of a choice-signalling meaning to this motor action.

Together, these data support a model that acetylcholine release in the sensory cortex is driven by directed motor actions to gather information (e.g. whisking) and act upon it (e.g. licking). This contrasts from reports that acetylcholine release is driven by reinforcement-predictive cues 11,12 , 
16 and reward itself 11,12 . This difference may be because the auditory cue in our task does not predict reward, only the temporal availability of a stimulus. In contrast to passive cue-reward association, our task requires active exploration to gather reward-predictive information, revealing a motor requirement for acetylcholine release. Our results also suggest that cue-induced changes in motor behavior (e.g. increased whisking, sniffing or anticipatory licking) and other orofacial movements $\underline{31}$ may also provide a meaningful contribution to acetylcholine release in passive association tasks.

The lack of reward-evoked acetylcholine release in S1 may be due to several factors. One possibility is that reward activity in BF is transmitted only to particular targets such as BLA 11 , but not $\mathrm{S} 1$, due to projection specificity in subpopulations of BF cholinergic neurons $\stackrel{7}{\text {. Second, while }}$ reward-delivery signals have been observed in cholinergic neurons of HDB and NB ${ }^{27}$, fiber photometry in those areas show reward-associated transients are small relative to lick-evoked transients and tightly correlated with an increase of lick rate following water delivery ${ }^{21}$. Perhaps reward-delivery only indirectly drives acetylcholine release via changes in licking patterns. However, this result may depend on task and reward structure. Tasks with variable reward probability have shown decreased cholinergic activity to highly likely rewards $\underline{12} \underset{27}{2}$. In our task, licking on go trials guaranteed reward, which may have shifted reward-delivery activity earlier to the time of the choice-signaling lick, when reward becomes expected.

Intriguingly, the acetylcholine release associated with the first lick began ramping several hundred milliseconds prior to lick detection (Figure 4C). This may be due to a combination of following factors: whisking precedes licking and drives modest and additive acetylcholine release (Figure 3 ), decision related activity precedes motor action by some amount of time $\underline{39}$, and the tongue requires about $150-200 \mathrm{~ms}$ from protrusion initiation to lickport contact ${ }^{32}$. These are partially counterbalanced by the indicator rise time, which is dependent on the underlying acetylcholine concentration profile and likely on the order of tens of milliseconds for a few percent $\Delta \mathrm{F} / \mathrm{F}$ change ${ }^{30}$. Thus, we must consider the possibility that some of the first lick-associated acetylcholine dynamics are caused by an internal choice deliberation, rather than the initiation of motor action. This possibility is reinforced by the observation that onset of acetylcholine release is more closely aligned to first lick on early than on expert sessions

(Figure 6A), suggestive of expert-specific choice-associated dynamics overlaid on motor-triggered dynamics common to both session classes.

The patterns of acetylcholine release in response to whisking and licking suggest potential functions for acetylcholine on sensory cortical circuitry ${ }^{40,41}$. VIP cells in S1 are activated by whisking $\underline{42}$ via acetylcholine release $\underline{43}$ leading to disinhibition of excitatory neuron dendrites where top-down contextual information arrives in $\mathbf{S 1}$ 44, 45, 46. Thus, whisking-induced acetylcholine release could enhance integration of contextual information with sensory input in S1 neurons, providing, for example, a potential mechanism for combining motor and touch

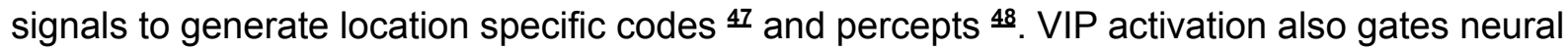
plasticity in cortex $\stackrel{49}{ }$. Higher task performance is associated with increased acetylcholine in V1 26. Our similar results in S1 (Figure 6B) identified that this increase is specific to the first lick and persists for several seconds (Figure 4). Thus, this increase is well-poised to provide windows of 
enhanced neural plasticity via VIP cell activation while the consequences of decisions are evaluated.

This work is only a step towards understanding the function of acetylcholine dynamics in cortex and sensory processing. We did not determine the extent to which the S1 acetylcholine dynamics reflect activity from nucleus basalis (NB) versus horizontal diagonal band (HDB) or their subdivisions which project to $S 1 \mathrm{I}$. An intriguing possibility is that the selective potentiation of choice-signalling action may be dissociable by afferent source. Our observations were made from pooled fluorescence responses of GRABs expressed across all neurons. The specificity of acetylcholine action on particular cortical cell types (e.g. VIP-expressing interneurons) raises the question of whether this reflects differing patterns of receptor expression $\underline{\mathbf{5 0}}$ or also involves preferential targeting by cholinergic axons. Finally, while we quantified the triggers and timescales of acetylcholine dynamics on cortical targets, substantial additional work is required to determine the functional consequences of those time limits. Manipulation of local acetylcholine dynamics and cellular targets at specific moments during task performance and acquisition could clarify acetylcholine's potential roles in regulation of sensory integration and cortical plasticity. 


\section{Methods}

Key Resources Table

\begin{tabular}{|c|c|c|}
\hline Reagent or Resources & Sources & Identifier \\
\hline \multicolumn{3}{|l|}{ Virus } \\
\hline AAV-GRAB-ACh3.0 & Vigene & \\
\hline \multicolumn{3}{|c|}{ Experimental Models: Organisms/Strains } \\
\hline Mouse: C57BL/6J & Jackson Laboratory & RRID:IMSR_JAX:00064 \\
\hline \multicolumn{3}{|l|}{ Software and Algorithms } \\
\hline Matlab & Math Works & 2018B, 2021a \\
\hline Scanbox & Dario Ringach, UCLA & https://scanbox.org \\
\hline BControl & Carlos Brody & $\begin{array}{l}\text { https://brodylabwiki.princeton. } \\
\text { edu/bcontrol/index.php?title= } \\
\text { Main_Page }\end{array}$ \\
\hline \multicolumn{3}{|l|}{ Other } \\
\hline Two-photon microscope & Neurolabware & \\
\hline $\begin{array}{l}\text { NIR laser ( } 2 \text { photon } \\
\text { microscope) }\end{array}$ & Spectra-Physics & InSight DS+ \\
\hline StreamPix & Norpix & RRID:SCR_015773 \\
\hline Pneumatic slide & Festo & Cat\#170496 \\
\hline Solenoid valve & Lee Company & Cat\#acA800-500um \\
\hline CMOS camera & Basler & Cat\#acA800-500um \\
\hline Telecentric lens & Edmund optics & Cat\#58-259 \\
\hline
\end{tabular}

\section{Experimental Model and Subject Details}

\section{Animals}

We used 2.5-4 months old male $(n=2)$ and female $(n=6)$ C57BL/6J mice (\#000664, The Jackson Laboratory). Mice were maintained on a 12:12 reversed light-dark cycle. After water restriction, health status was assessed everyday following a previously reported guideline (Guo et al., 2014). All procedures were performed in accordance with the University of Southern California Institutional Animal Care and Use Committee Protocol 20732 and 20788. 


\section{Method Details \\ Headbar surgeries}

Before each surgery, rimadyl tablet was given $0.5 \mathrm{mg} /$ tablet 24 hours before surgery. buprenorphine-SR and marcaine were injected subcutaneously at $0.5 \mathrm{mg} / \mathrm{kg}$ and $2 \%$ right before the surgery. A customized stainless steel headbar was attached to the skull using layers of Krazy glue (Elmer's Products, Inc) and dental cement (Lang Dental Mfg. Co., Inc).

\section{Intrinsic signal imaging}

Intrinsic signal imaging (ISI) was done 3 days after headbar surgery and 7 days after the cranial window surgery. All whiskers except the C2 whisker were trimmed before ISI. To identify C2 whisker, C2 whisker was stimulated using a Piezo stimulator when mice were under light isoflurane anesthesia (0.8-1.0\%). Comparisons of acetylcholine dynamics inside the C2 column versus surround were based on column-sized hand-drawn ROls centered on the ISI hotspot.

\section{Cranial window and virus injection surgeries}

In all mice C57BL/6J mice ( $\mathrm{n}=8$ ), AAV9-hSyn-GRAB-ACh3.0 (Addgene Plasmid \#121922 https://www.addgene.org/121922/ ) was injected from 1x aliquots during the cranial window installation. A glass capillary (Wiretrol $®$ II, Drummond) was pulled to $10-20 \mu \mathrm{m}$ in tip diameter using a micropipette puller (Model P-97, Sutter instrument), and tip beveled to about 30 degrees. The glass window was $2 \times 2 \mathrm{~mm}$ glass hand fused to $3 \times 3$ glass (both $0.13-0.17 \mathrm{~mm}$ thickness) with ultraviolet curing glue (Norland optical adhesive 61, Norland Inc.).

Before each surgery, rimadyl tablet was given $0.5 \mathrm{mg} /$ tablet 24 hours prior and Buprenorphine-SR was injected subcutaneously at $0.5 \mathrm{mg} / \mathrm{kg}$ immediately before. A 2x2 mm square of skull whose center was the identified C2 whisker barrel region was removed. Virus was backfilled into a pipette of mineral oil (M5904, Sigma-Aldrich). We injected 400nl virus into the identified C2 barrel column through a single injection site over $4 \mathrm{~min}$ at $300 \mu \mathrm{m}$ below pia, withdrawing after an additional two minutes. The exposed brain region was then covered with the home-made glass window. Targeting of the C2 column was confirmed via ISI one week after cranial window surgery, after which water restriction commenced.

\section{Behavioral task and training}

Mice were trained in a whisker-guided Go/No-go localization discrimination task (O'Connor et al., 2010). During training, a smooth black pole with $0.6 \mathrm{~mm}$ diameter (a plunger for glass capillary, Wiretrol $\mathbb{I}^{\mathrm{II}}$, painted with black lubricant, industrial graphite dry lubricant, the B'laster Corp.) was vertically presented at two positions using a pneumatic slide (Festo), with the posterior position rewarded (Go trials) and anterior position non-rewarded (No-go trials). The pole came into touch range within $100 \mathrm{~ms}$ of pole motion onset. Mice used a single whisker (C2) to discriminate positions. Mice indicated their decision through licking or withhold licking during the answer period according to pole position. Licks in the 1s sampling period were ignored. On Hit trials, mice received water rewards on the first lick in the 1s answer period. On False Alarm trials, based on each mouse's learning process, each lick during the answer period re-triggered a timeout that lasted 0-4 seconds. Miss and Correct Rejection trials were unpunished. The 
behavioral task was controlled by MATLAB-based BControl software (C. Brody, Princeton University).

We trained mice in a stepwise manner. First, we associated the timing of cue and pole to reward to let mice learn that water can come out of the lick port and the water is temporally associated with a pole presentation. Mice usually learned the association in a few minutes. Then we introduced Go trials only to let the mice learn the trial structure, which usually took 1-2 sessions for the mice to achieve high performance. After the mice were able to get over 10 Hit trials in a row, we introduced No-go trials. During early training, we adjusted the No-go trials probability and time-out time on False Alarm trials to help mice learn, settling on 50\% No-G- probability and 0 s timeout once mice did not get discouraged and stop licking after a series of misses. Expert threshold was set at $>70 \%$ accuracy continuously for 3 sessions. After the animals became experts, we trimmed the animal's last whisker (C2 whisker) to test whether the mice learned the task in a whisker-dependent manner.

\section{Whisker motion acquisition and analysis}

Backlit whisker motion video was acquired with a CMOS camera (Basler acA800-500um), StreamPix Software (NorPix Inc.) at $311 \mathrm{~Hz}$ through telecentric lens $\left(0.09 X_{1} / 2\right.$ " GoldTL TM \#58-259, Edmund optics), a CMOS camera (Basler acA800-500um). to record whisker motion. Camera frames were triggered and synced by BControl and Ardunio. We tracked whisker position with the Janelia Whisker Tracker

(https://www.janelia.org/open-science/whisk-whisker-tracking, Clack et al., 2012). The fur was masked improve tracking quality. The whisker's azimuthal angle was calculated at the intersection of the mask and whisker trace. Whisking midpoint, amplitude and phase was decomposed from this angle using the Hilbert transform, as described in Cheung et al., 2019.

\section{Two-photon microscopy}

The two-photon microscope (Neurolabware) used a galvanometer scanner $(6215 \mathrm{H}$, Cambridge Technology), Pockels cell (350-105-02 KD*P EO modulator, Conoptics), a resonant scanner (CRS8, Cambridge Technology), an objective (W Plan-Apochromat 20×/1.0, Zeiss), a GaAsP photomultiplier tube (H10770B-40, Hamamatsu), and a $510 \mathrm{~nm}$ emission filter (FF01-510/84-50, Semrock). We used an $80 \mathrm{MHz}$ tunable laser at $940 \mathrm{~nm}$ (Insight DS+, Spectra-Physics) for GRAB-Ach3.0 excitation. Imaging was continuous throughout each session. The scope was controlled by a MATLAB-based software Scanbox with custom modifications. Imaging frequency was $15.44 \mathrm{frames} / \mathrm{s}$ on the size of the FOV $(512 \times 796)$. Spatial resolution was $1.4 \mu \mathrm{m}$ per pixel.

\section{Data analysis and statistics}

All imaging data were processed in Matlab. We excluded the first 100s for each session due to non-linear photodynamics which stabilized after 100 seconds of continuous excitation scanning (SFig2). In Figure 2B we used the mean fluorescence intensity of the sample session as baseline. In Figure 2C-2E we used 16 frames after trial start as baseline. Figure 3A and 3B we used 1 second before stimuli (pole onset and pole withdrawal) as baseline). Figure 3D we used 16 frames after trial start as baseline. Figure 4A, 4B, 5A and 6A we used 32 frames before first 
bioRxiv preprint doi: https://doi.org/10.1101/2021.12.21.473699; this version posted December 23, 2021. The copyright holder for this preprint (which was not certified by peer review) is the author/funder. All rights reserved. No reuse allowed without permission.

lick to 16 frames before first lick as baseline. Statistical comparisons were made using paired T Test corrected for multiple comparisons. 


\section{References}

1. Donoghue, John P., and Kristen L. Carroll. "Cholinergic modulation of sensory responses in rat primary somatic sensory cortex." Brain research 408.1-2 (1987): 367-371.

2. Tremblay, Nicole, Richard A. Warren, and Robert W. Dykes. "Electrophysiological studies of acetylcholine and the role of the basal forebrain in the somatosensory cortex of the cat. II. Cortical neurons excited by somatic stimuli." Journal of neurophysiology 64.4 (1990): 1212-1222.

3. Hars, Bernard, et al. "Basal forebrain stimulation facilitates tone-evoked responses in the auditory cortex of awake rat." Neuroscience 56.1 (1993): 61-74.

4. Goard, Michael, and Yang Dan. "Basal forebrain activation enhances cortical coding of natural scenes." Nature neuroscience 12.11 (2009): 1444-1449.

5. Parikh, Vinay, et al. "Prefrontal acetylcholine release controls cue detection on multiple timescales." Neuron 56.1 (2007): 141-154.

6. Zhang, Hao, Shih-Chieh Lin, and Miguel AL Nicolelis. "A distinctive subpopulation of medial septal slow-firing neurons promote hippocampal activation and theta oscillations." Journal of neurophysiology 106.5 (2011): 2749-2763.

7. Kim, Jae-Hyun, et al. "Selectivity of neuromodulatory projections from the basal forebrain and locus ceruleus to primary sensory cortices." Journal of Neuroscience 36.19 (2016): 5314-5327.

8. Reimer, Jacob, et al. "Pupil fluctuations track rapid changes in adrenergic and cholinergic activity in cortex." Nature communications 7.1 (2016): 1-7.

9. Chubykin, Alexander A., et al. "A cholinergic mechanism for reward timing within primary visual cortex." Neuron 77.4 (2013): 723-735.

10. Liu, Cheng-Hang, et al. "Selective activation of a putative reinforcement signal conditions cued interval timing in primary visual cortex." Current Biology 25.12 (2015): 1551-1561.

11. Crouse, Richard B., et al. "Acetylcholine is released in the basolateral amygdala in response to predictors of reward and enhances the learning of cue-reward contingency." Elife 9 (2020): e57335.

12. Sturgill, James Fitzhugh, et al. "Basal Forebrain-Derived Acetylcholine Encodes Valence-Free Reinforcement Prediction Error." BioRxiv, Feb. 2020 13. Kilgard, Michael P., and Michael M. Merzenich. "Cortical map reorganization enabled by nucleus basalis activity." Science 279.5357 (1998): 1714-1718.

14. Froemke, Robert C., et al. "Long-term modification of cortical synapses improves sensory perception." Nature neuroscience 16.1 (2013): 79-88.

15. Jiang, $\mathrm{Li}$, et al. "Cholinergic signaling controls conditioned fear behaviors and enhances plasticity of cortical-amygdala circuits." Neuron 90.5 (2016): 1057-1070. 
16. Guo, Wei, Blaise Robert, and Daniel B. Polley. "The cholinergic basal forebrain links auditory stimuli with delayed reinforcement to support learning." Neuron 103.6 (2019): 1164-1177.

17. Hasselmo, Michael E., and Martin Sarter. "Modes and models of forebrain cholinergic neuromodulation of cognition." Neuropsychopharmacology 36.1 (2011): 52-73.

18. Lee, Seung-Hee, and Yang Dan. "Neuromodulation of brain states." Neuron 76.1 (2012): 209-222.

19. Zaborszky, Laszlo, et al. "Neurons in the basal forebrain project to the cortex in a complex topographic organization that reflects corticocortical connectivity patterns: an experimental study based on retrograde tracing and 3D reconstruction." Cerebral cortex 25.1 (2015): 118-137.

20. Li, Xiangning, et al. "Generation of a whole-brain atlas for the cholinergic system and mesoscopic projectome analysis of basal forebrain cholinergic neurons." Proceedings of the National Academy of Sciences 115.2 (2018): 415-420. 21. Robert, Blaise, et al. "A functional topography within the cholinergic basal forebrain for processing sensory cues associated with reward and punishment." bioRxiv (2021). 22. Inglis, F. M., and H. C. Fibiger. "Increases in hippocampal and frontal cortical acetylcholine release associated with presentation of sensory stimuli." Neuroscience 66.1 (1995): 81-86.

23. Eggermann, Emmanuel, et al. "Cholinergic signals in mouse barrel cortex during active whisker sensing." Cell reports 9.5 (2014): 1654-1660.

24. Chaves-Coira, Irene, et al. "Basal forebrain nuclei display distinct projecting pathways and functional circuits to sensory primary and prefrontal cortices in the rat." Frontiers in neuroanatomy 12 (2018): 69.

25. Herrero, Jose L., et al. "Acetylcholine contributes through muscarinic receptors to attentional modulation in V1." Nature 454.7208 (2008): 1110-1114.

26. Pinto, Lucas, et al. "Fast modulation of visual perception by basal forebrain cholinergic neurons." Nature neuroscience 16.12 (2013): 1857-1863.

27. Hangya, Balázs, et al. "Central cholinergic neurons are rapidly recruited by reinforcement feedback." Cell 162.5 (2015): 1155-1168.

28. Harrison, Thomas C., et al. "Calcium imaging of basal forebrain activity during innate and learned behaviors." Frontiers in neural circuits 10 (2016): 36.

29. Richardson, Russell T., and M. R. DeLong. "Context-dependent responses of primate nucleus basalis neurons in a go/no-go task." Journal of Neuroscience 10.8 (1990): 2528-2540.

30. Jing, Miao, et al. "An optimized acetylcholine sensor for monitoring in vivo cholinergic activity." Nature methods 17.11 (2020): 1139-1146.

31. Lohani, Sweyta, et al. "Dual color mesoscopic imaging reveals spatiotemporally heterogeneous coordination of cholinergic and neocortical activity." bioRxiv (2020). 
32. Bollu, Tejapratap, et al. "Cortex-dependent corrections as the tongue reaches for and misses targets." Nature 594.7861 (2021): 82-87.

33. Kim, Jinho, et al. "Behavioral and Neural Bases of Tactile Shape Discrimination Learning in Head-Fixed Mice." Neuron 108.5 (2020): 953-967.

34. Guo, Zengcai V., et al. "Procedures for behavioral experiments in head-fixed mice." PloS one 9.2 (2014): e88678.

35. Klinkenberg, Inge, Anke Sambeth, and Arjan Blokland. "Acetylcholine and attention." Behavioural brain research 221.2 (2011): 430-442.

36. McGaughy, Jill, et al. "Selective behavioral and neurochemical effects of cholinergic lesions produced by intrabasalis infusions of 192 lgG-saporin on attentional performance in a five-choice serial reaction time task." Journal of Neuroscience 22.5 (2002): 1905-1913.

37. Kuchibhotla, Kishore V., et al. "Parallel processing by cortical inhibition enables context-dependent behavior." Nature neuroscience 20.1 (2017): 62-71.

38. O'Connor, Daniel H., et al. "Neural activity in barrel cortex underlying vibrissa-based object localization in mice." Neuron 67.6 (2010): 1048-1061.

39. Shadlen, Michael N., and William T. Newsome. "Motion perception: seeing and deciding." Proceedings of the national academy of sciences 93.2 (1996): 628-633.

40. Letzkus, Johannes J., et al. "A disinhibitory microcircuit for associative fear learning in the auditory cortex." Nature 480.7377 (2011): 331-335.

41. Urban-Ciecko, Joanna, et al. "Precisely timed nicotinic activation drives SST inhibition in neocortical circuits." Neuron 97.3 (2018): 611-625.

42. Lee, Soohyun, et al. "A disinhibitory circuit mediates motor integration in the somatosensory cortex." Nature neuroscience 16.11 (2013): 1662-1670.

43. Gasselin, Célia, et al. "Cell-type-specific nicotinic input disinhibits mouse barrel cortex during active sensing." Neuron 109.5 (2021): 778-787.

44. Takahashi, Naoya, et al. "Active cortical dendrites modulate perception." Science 354.6319 (2016): 1587-1590.

45. Takahashi, Naoya, et al. "Active dendritic currents gate descending cortical outputs in perception." Nature Neuroscience 23.10 (2020): 1277-1285.

46. Lacefield, Clay O., et al. "Reinforcement learning recruits somata and apical dendrites across layers of primary sensory cortex." Cell reports 26.8 (2019): 2000-2008. 47. Cheung, Jonathan Andrew, et al. "Independent representations of self-motion and object location in barrel cortex output." PLoS Biology 18.11 (2020): e3000882.

48. Cheung, Jonathan, et al. "The sensorimotor basis of whisker-guided anteroposterior object localization in head-fixed mice." Current Biology 29.18 (2019): 3029-3040.

49. Williams, Leena E., and Anthony Holtmaat. "Higher-order thalamocortical inputs gate synaptic long-term potentiation via disinhibition." Neuron 101.1 (2019): 91-102. 50. Yao, Zizhen, et al. "A taxonomy of transcriptomic cell types across the isocortex and hippocampal formation." Cell 184.12 (2021): 3222-3241. 
51. O'Connor, Daniel H., et al. "Vibrissa-based object localization in head-fixed mice." Journal of Neuroscience 30.5 (2010): 1947-1967. 52. Clack, Nathan G., et al. "Automated tracking of whiskers in videos of head fixed rodents." PLoS computational biology 8.7 (2012): e1002591. 


\section{Figure 1 - Learning of whisker-guided object location discrimination and associated motor actions.}

A
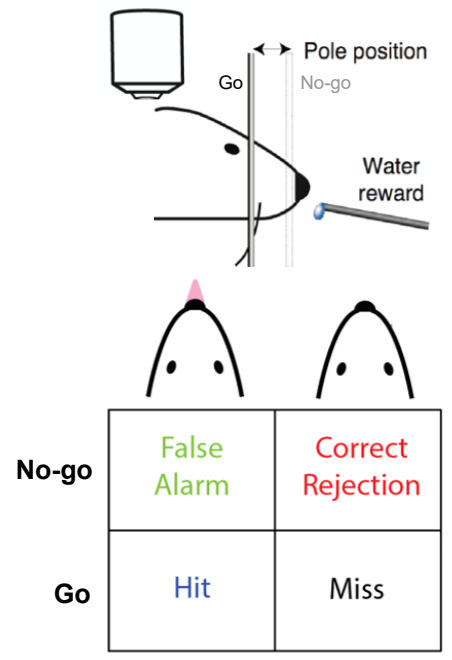

E

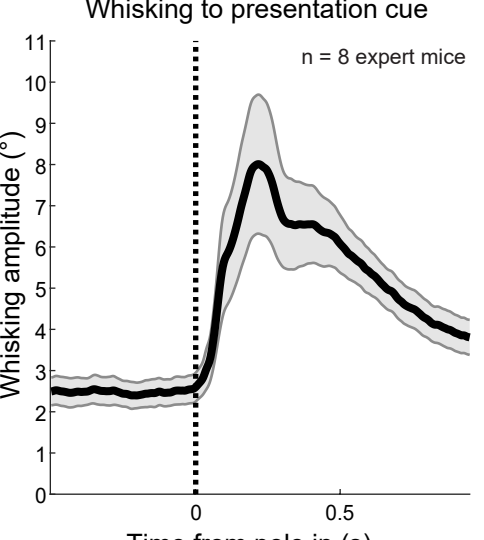

B

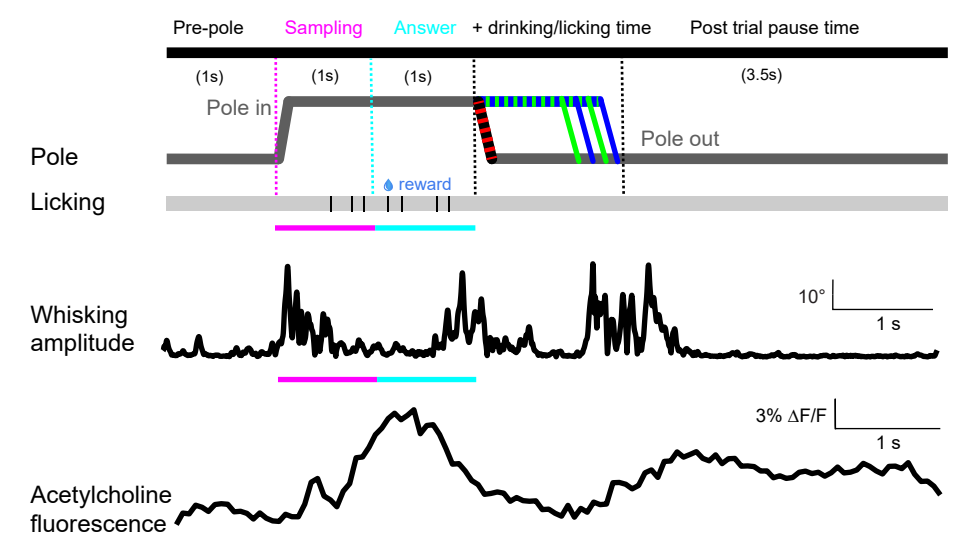

$\mathbf{F}$

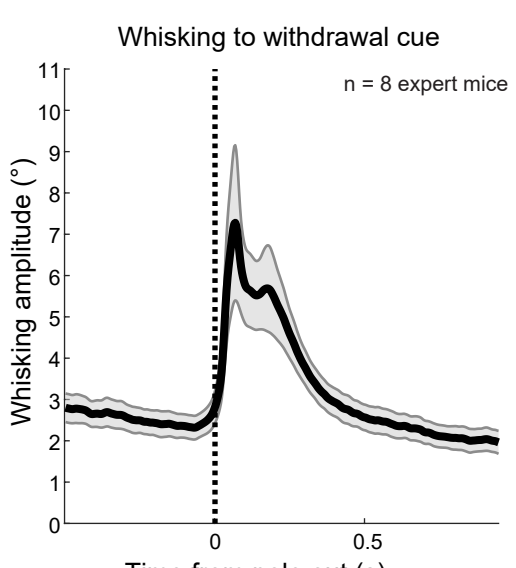

G
C
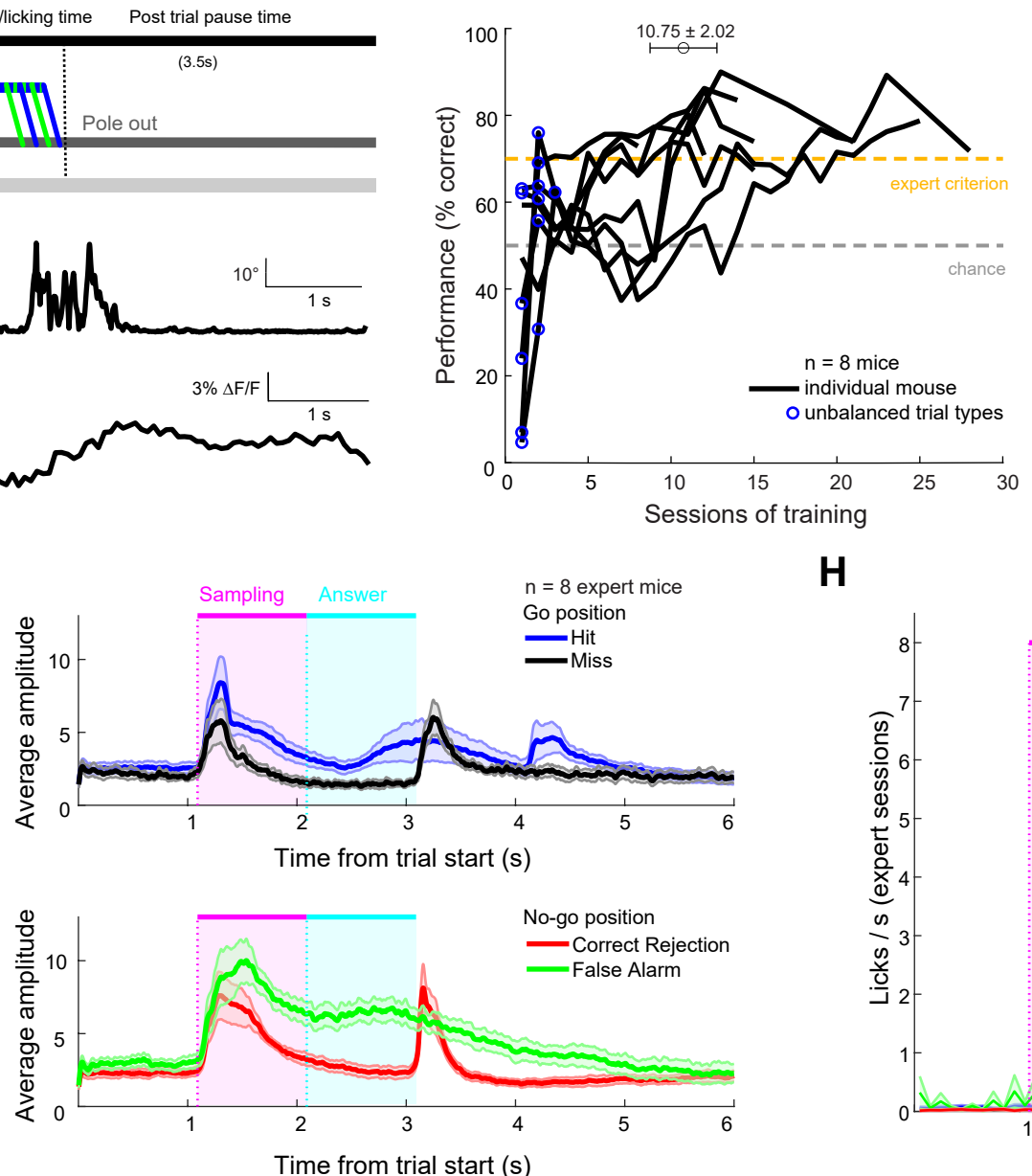

D

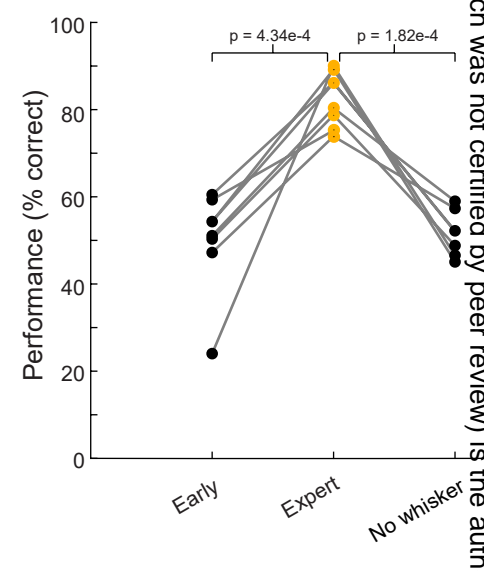

H

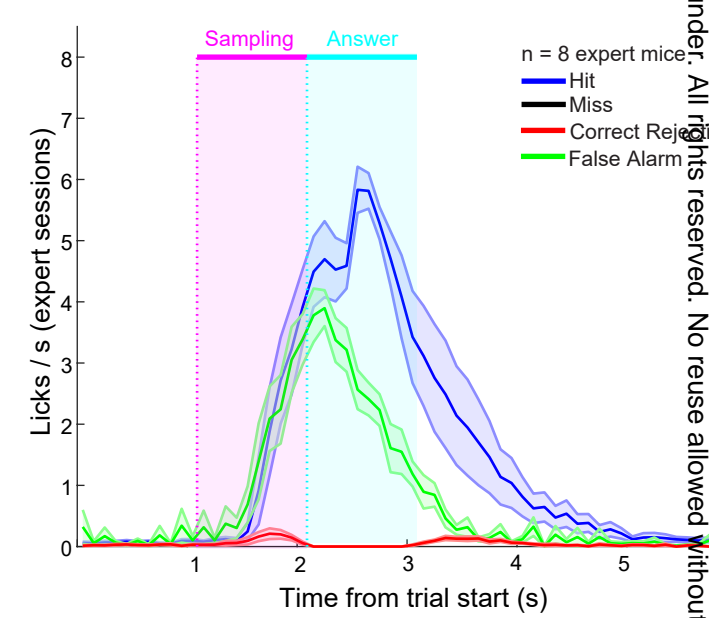

A.Two location discrimination task design with trial outcomes. B. Single trial structure with example licking, whisking, GRAB-ACh signal traces. C. Learning curves of 8 mice, mean \pm SEM sessions to expert. Blue circles, unbalanced go and no-go trials. Gold dash, expert threshold. Gray dash, chance level. D. Mean performance for three early and expert sessions, per mouse. 3 mice with 1 no-whisker session, 4 mice with 3 no-whisker sessions averaged. E. Average whisking amplitude aligned to pole in cue, 3 expert sessions / mouse. F. Same aligned to pole out cue. G. Grand mean trial averaged whisking amplitude by trial outcome, 3 expert sessions / mouse. $\mathbf{H}$. Same for licking rates. 
Figure 2 - Acetylcholine release in $\mathbf{S} 1$ varies with trial outch woutcome

A
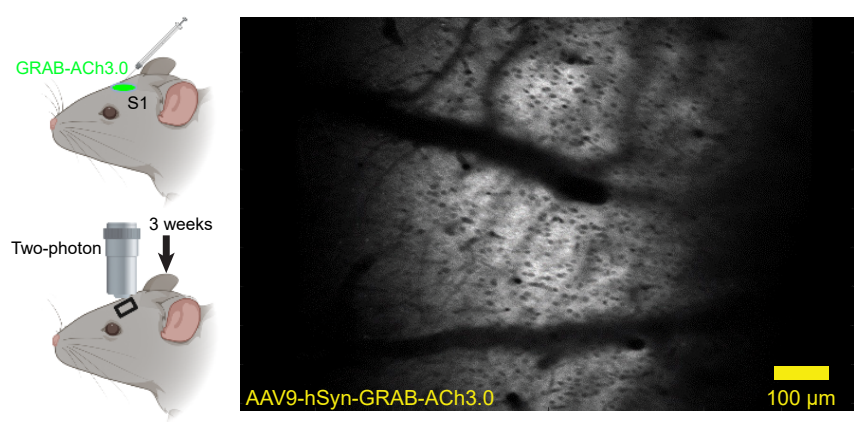

B

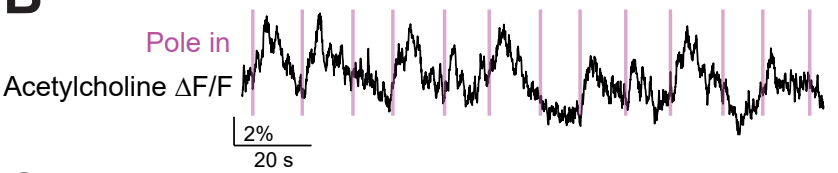

C

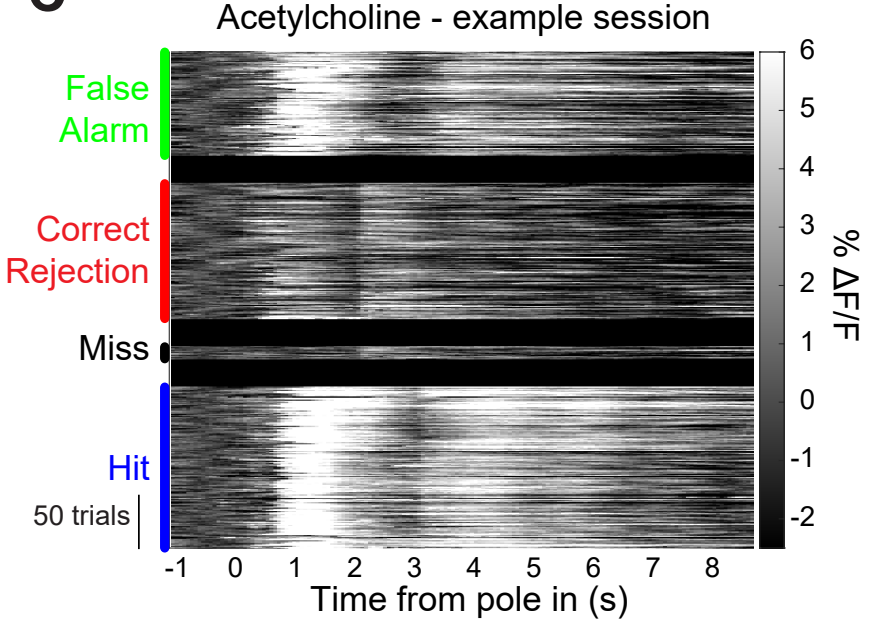

D Acetylcholine - mean expert sessions

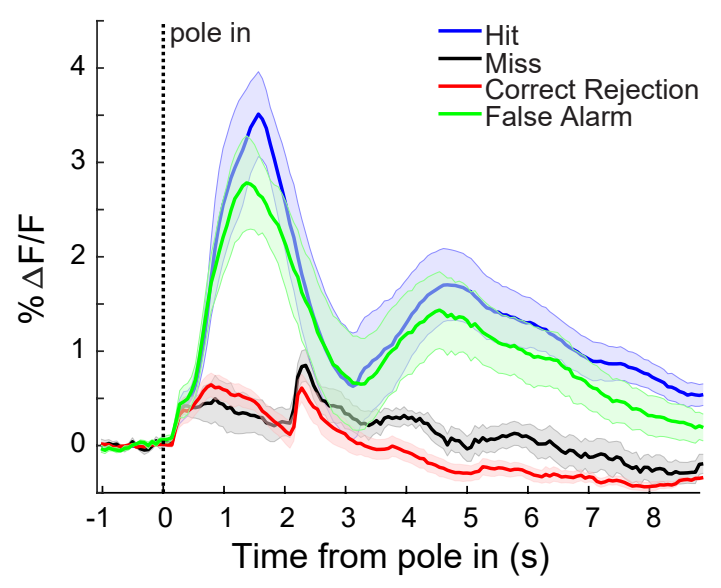

E Acetylcholine - spatial dynamics

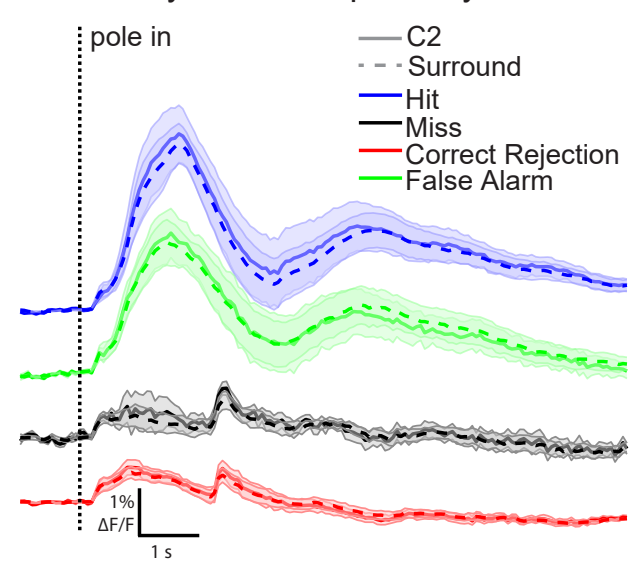

A. GRAB-ACh3.0 AAV expression in S1 barrel cortex, 3 weeks post injection. Cartoon generated by BioRender.com. B. Phasic increase of acetylcholine release after pole in cue in most trials. C. Acetylcholine induced fluorescence changes sorted by trial types, one expert example session. D. Grand average, 8 mice, 3 expert sessions each. E. Comparison of grand average acetylcholine dynamics within the C2 column (solid) and in the surround (dashed). 8 mice, 3 expert sessions each. 


\section{Figure 3 - Whisking drives acetylcholine release in S1}

A

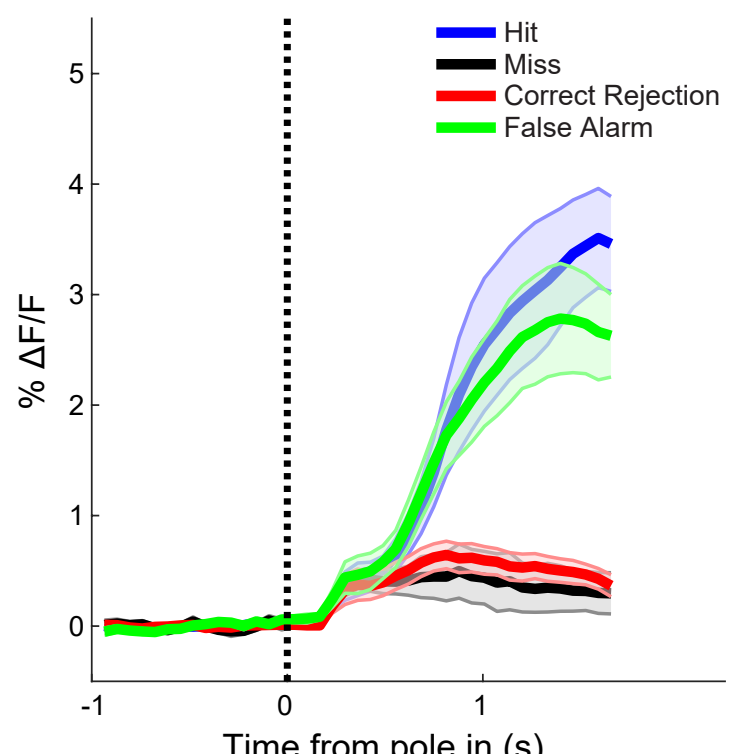

B

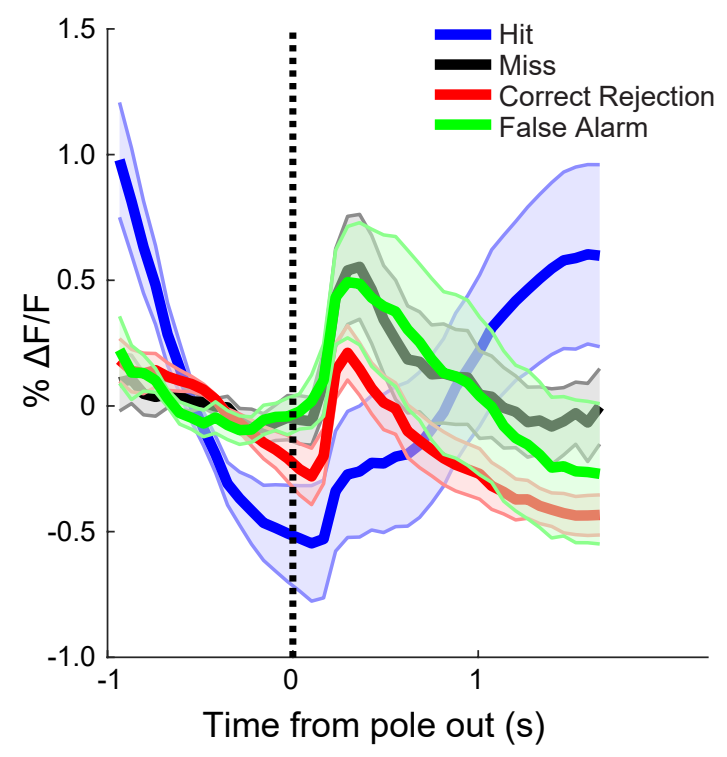

C

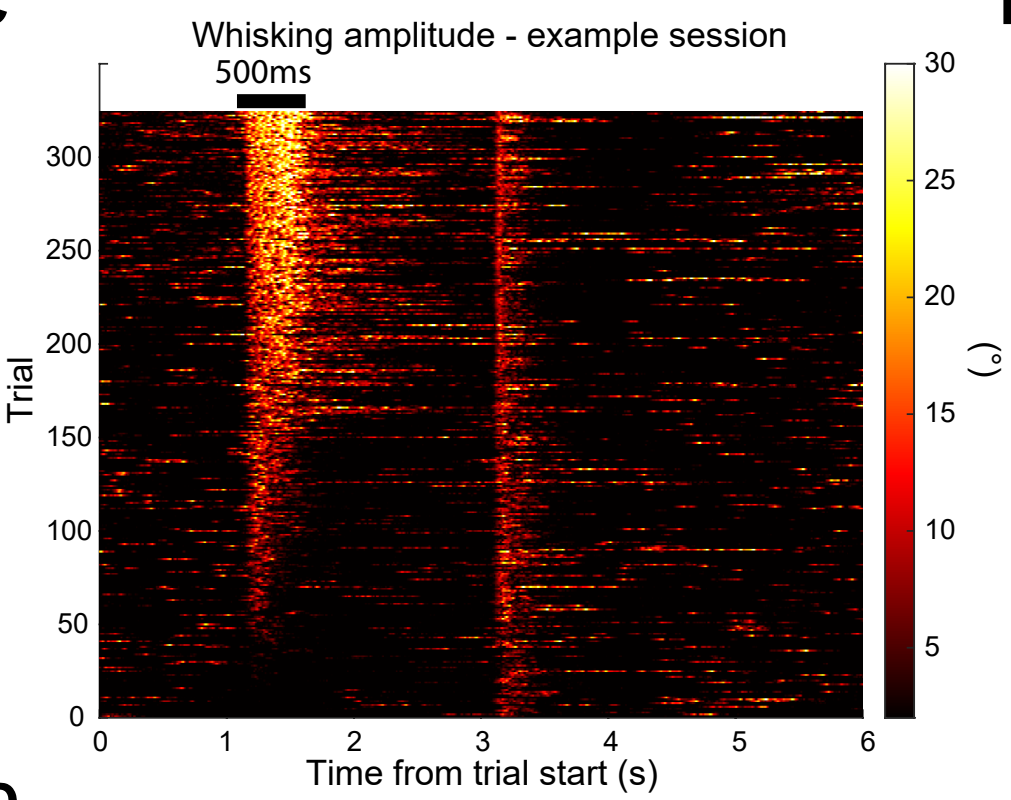

D

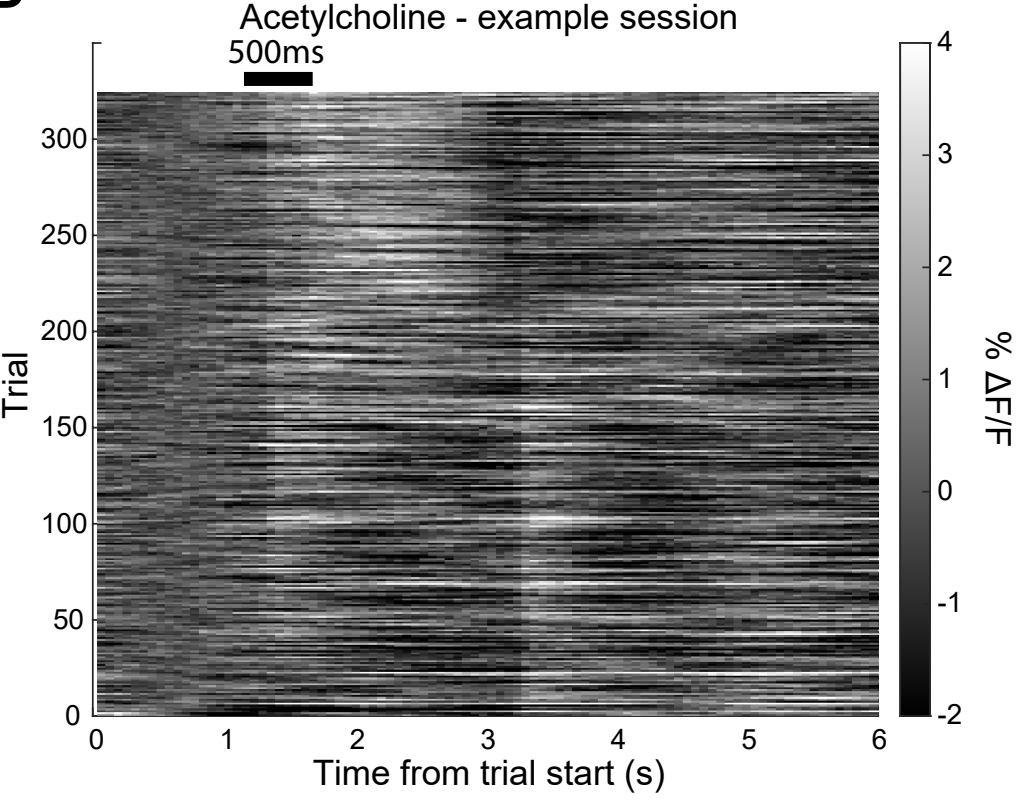

$\mathbf{E}$

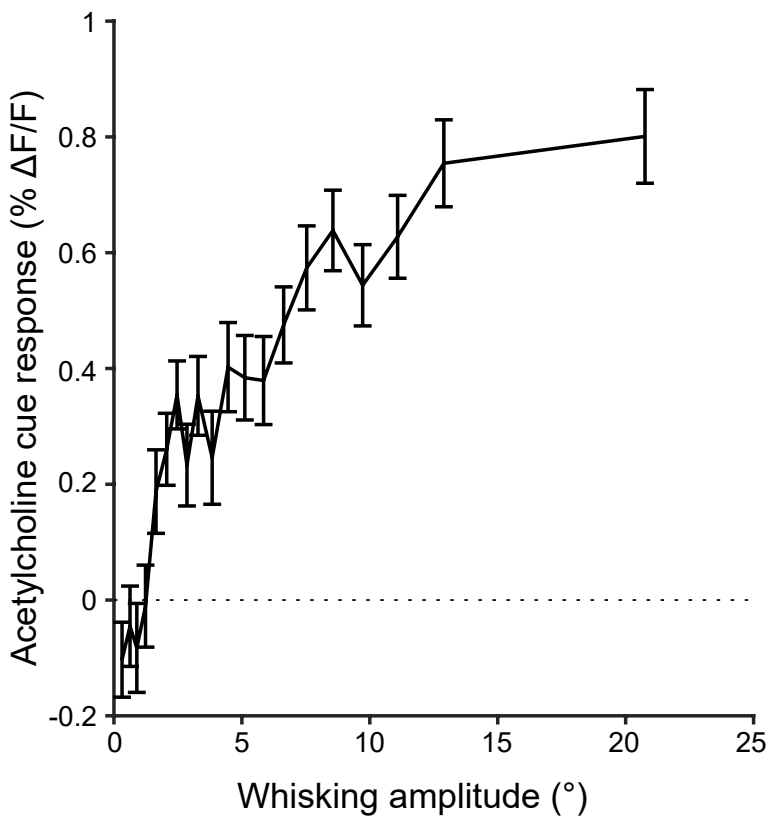

A. Grand mean acetylcholine fluorescence change aligned to pole in cue. 8 mice, 3 expert sessions each. B. Same to pole out cue. C. Whisking amplitude sorted by mean of $500 \mathrm{~ms}$ post pole in cue. No lick trials pooled from 3 expert sessions, 1 mouse. D. Same, for acetylcholine fluorescence change sorted by whisking amplitude. E. Grand mean of acetylcholine fluorescence change vs. whisking amplitude of $500 \mathrm{~ms}$ after pole in cue. No lick trials from 3 early and 3 expert sessions, 8 mice. 
Figure 4 - Licking strongly drives acetylcholine release in $\mathbf{S 1}$

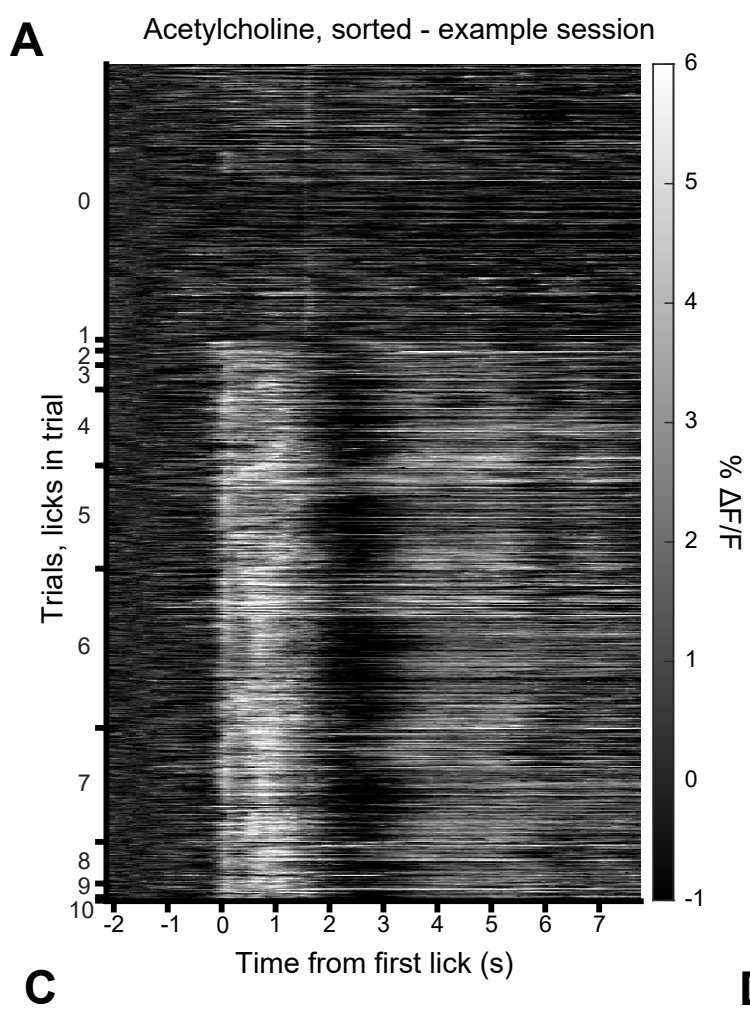

B

Licks, sorted - example session

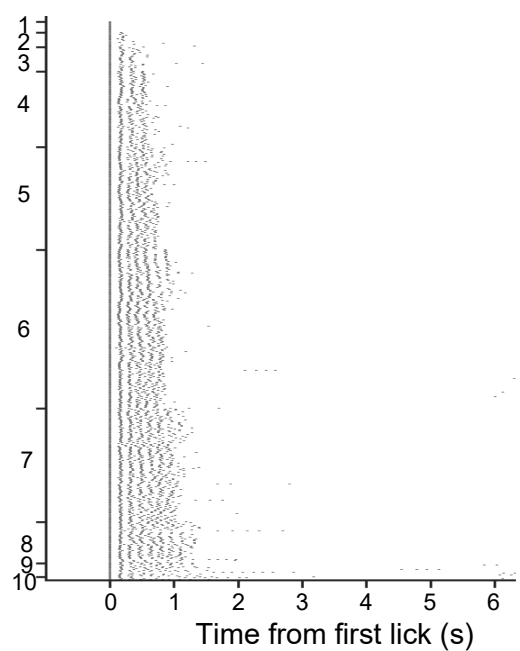

Lick interval distribution
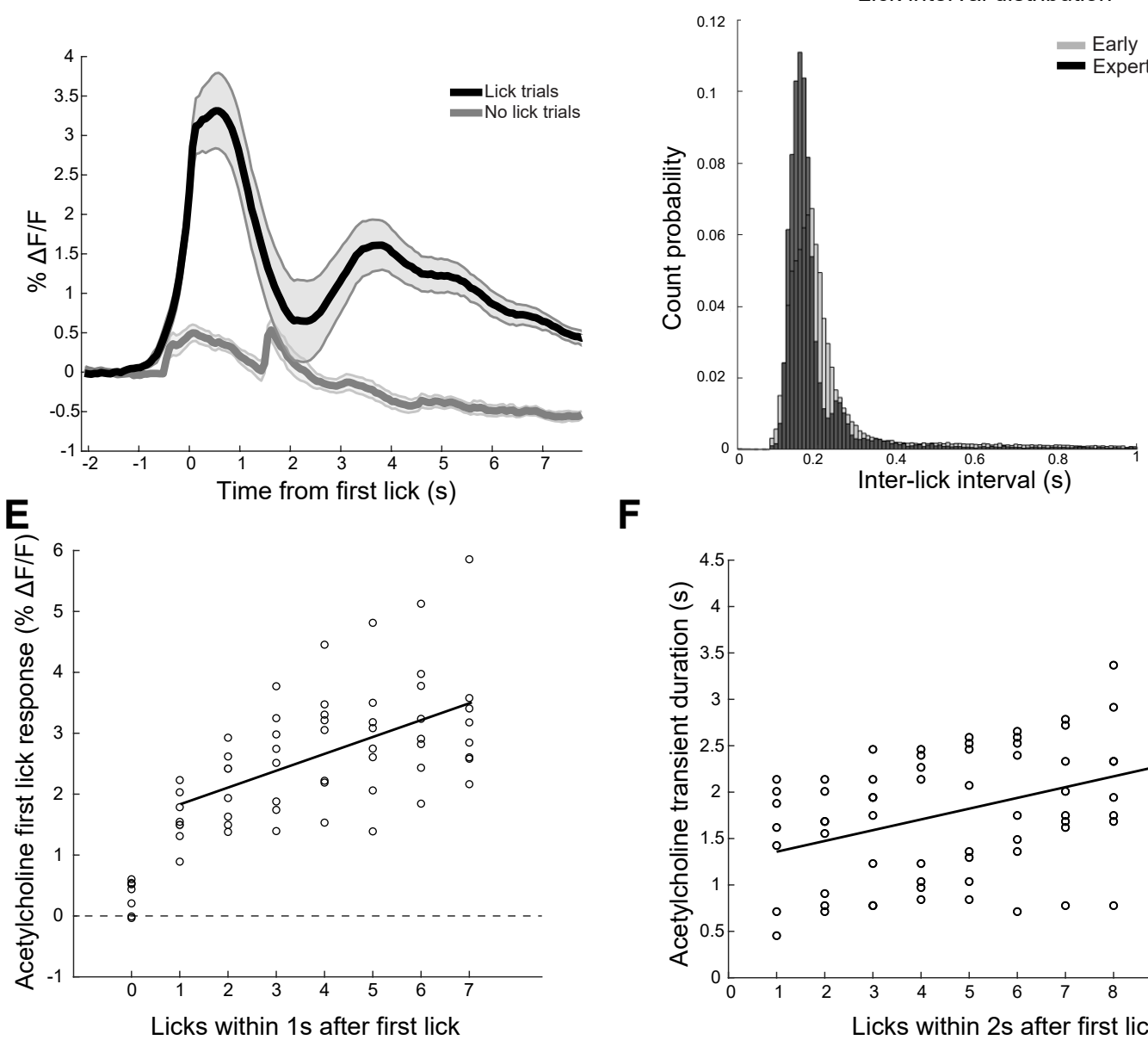

$\mathbf{F}$

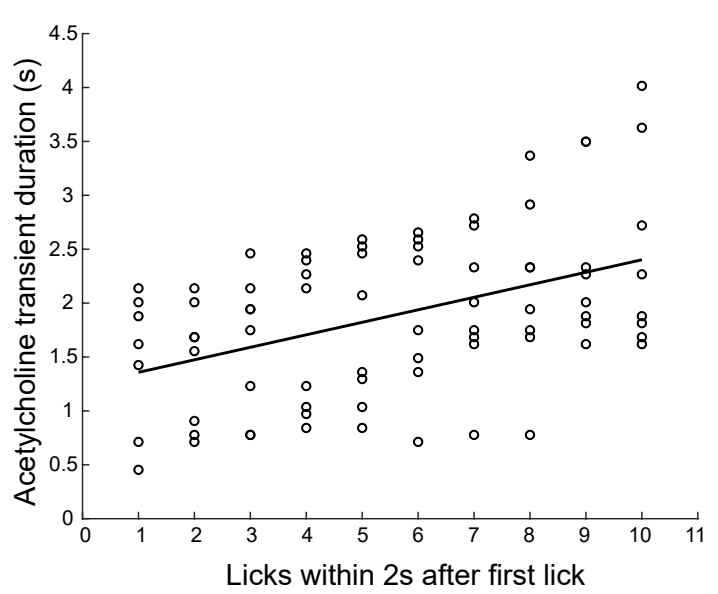

A. Acetylcholine fluorescence change across trials, sorted by number of licks in trial, aligned to first lick. One example expert session. B. Lick pattern across trials, sorted by number of licks in trial, aligned to first lick. Same example session as A. C. Grand mean acetylcholine fluorescence change aligned to first lick. 3 expert sessions, 8 mice. D. Inter-lick interval from early (gray) and expert (black) sessions. E. Mean acetylcholine fluorescence change in 1 second following first lick binned by number of licks in that period. Circles, mean 3 expert sessions / mouse. Linear fit from 1-7 licks. F. Duration, onset to trough, of the first acetylcholine transient, binned by number of licks within 2 seconds of first lick. Circles, mean 3 expert sessions / mouse. Linear fit from 1-10 licks. 


\section{Figure 5 - Reward delivery does not drive acetylcholine release in S1}

A
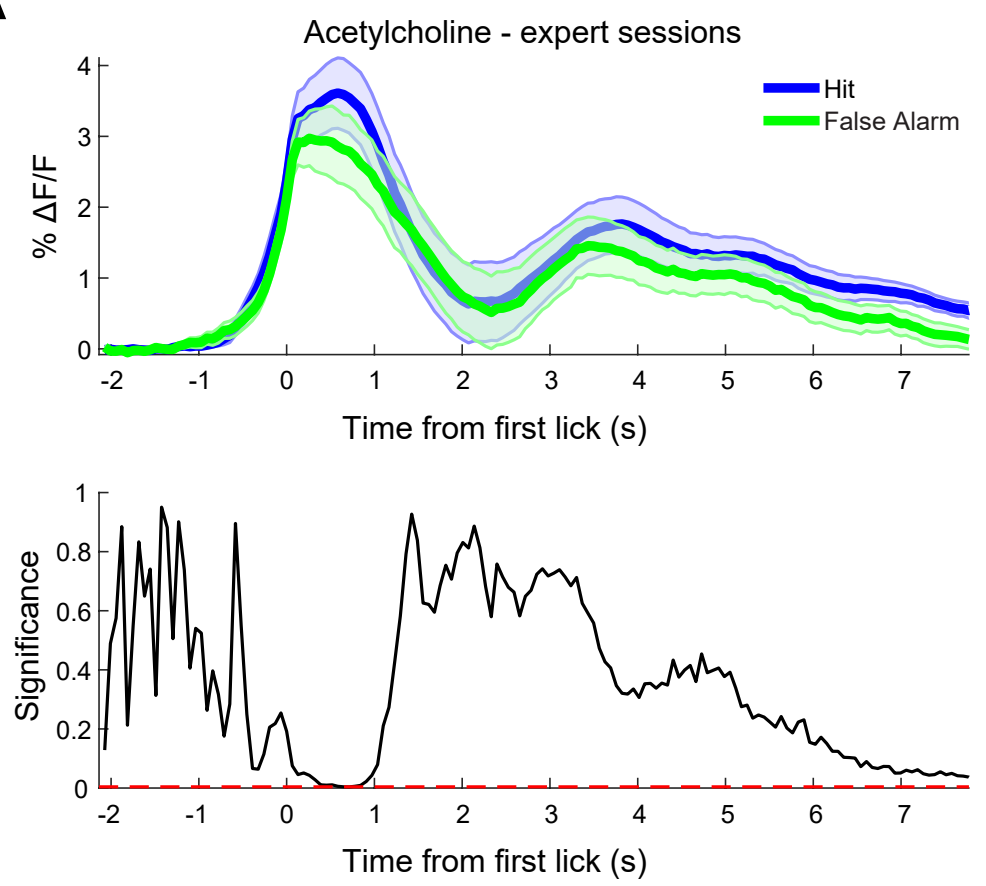

B

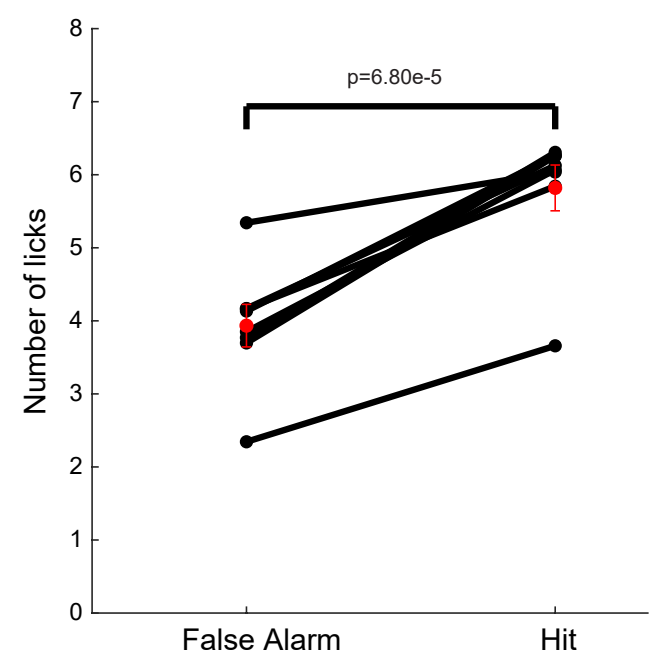

C

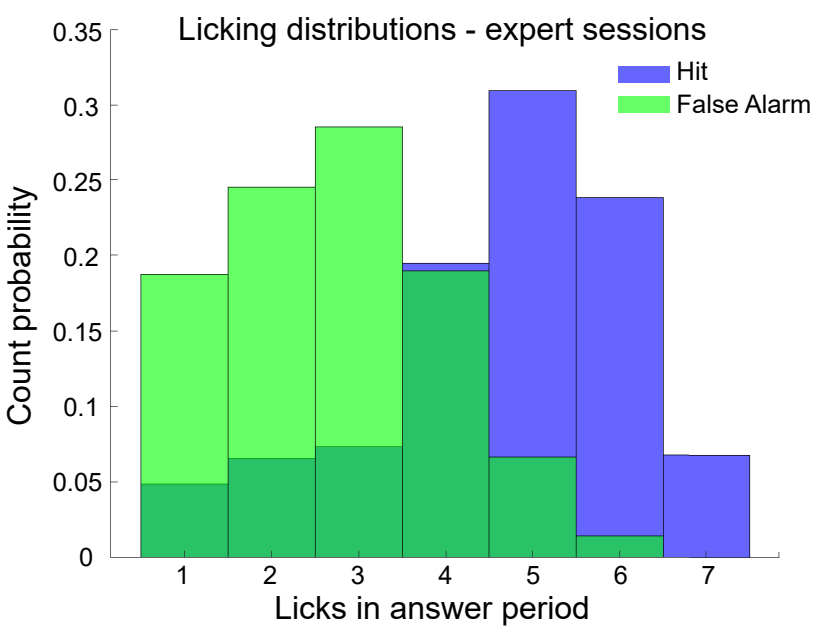

D
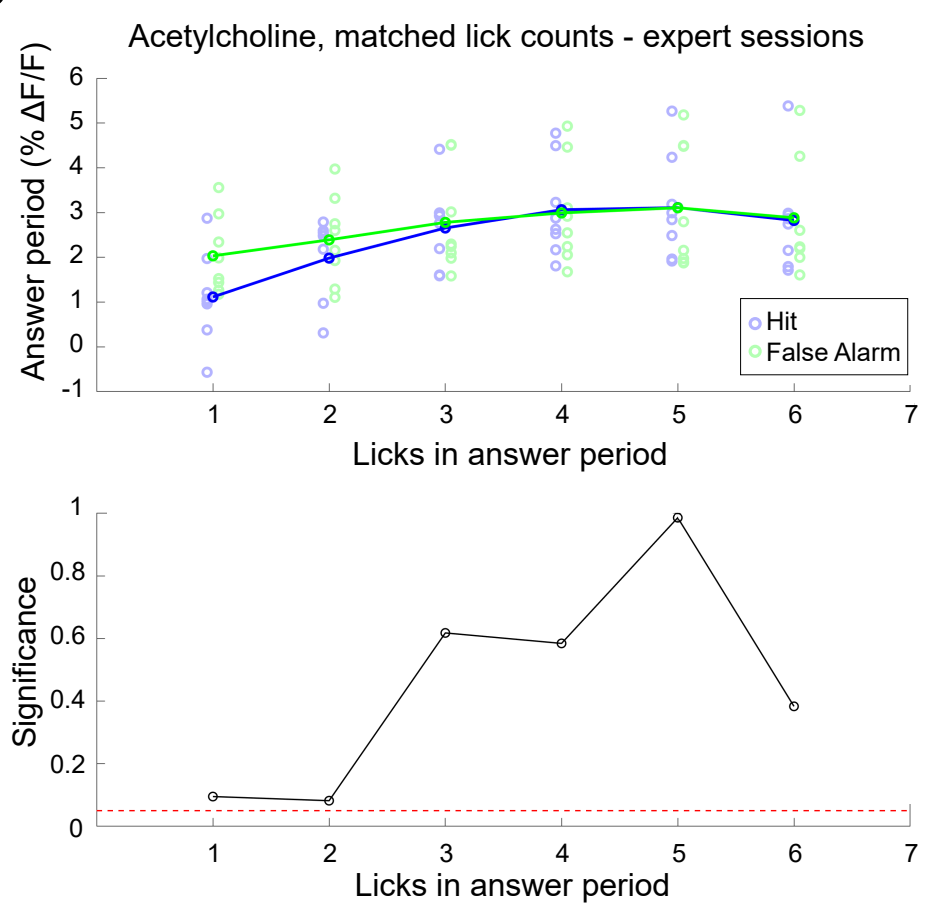

A.Top: Acetylcholine fluorescence change aligned to first lick from Hit (blue) and False Alarm (green) trials. Mean and SEM. Bottom: Significance test between Hit and False Alarm acetylcholine over time ( $p$-value, paired t-test, corrected for multiple comparisons). B. Mean number of licks in the answer period on Hit and False Alarm trials, 3 expert sessions per mouse. C. Distribution of lick counts in the answer period histogram for Hit (blue) and False Alarm (green) trials. D.Top: Mean acetylcholine fluorescence change in answer period, binned by licks in answer period for Hit (blue) and False Alarm (green). Bottom: Significance of difference between Hit and False Alarm trials, binned by licks ( $p$-value, paired t-test, corrected for multiple comparisons). 
Figure 6 Learning selectively potentiates acetylcholine release to choice-signalling licks in S1

A

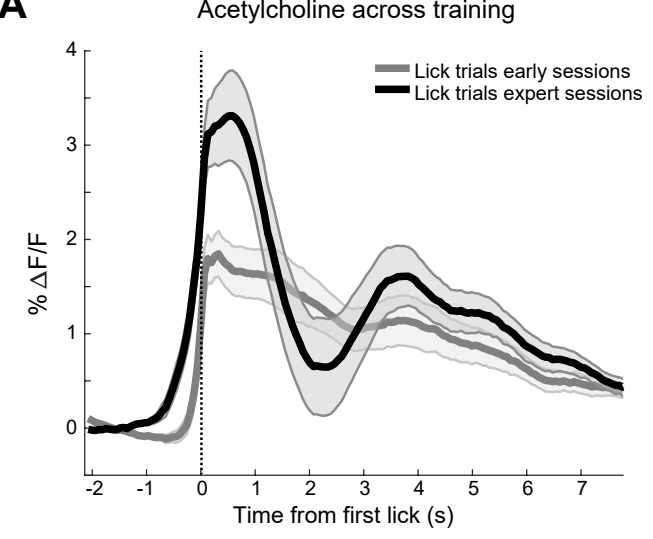

B

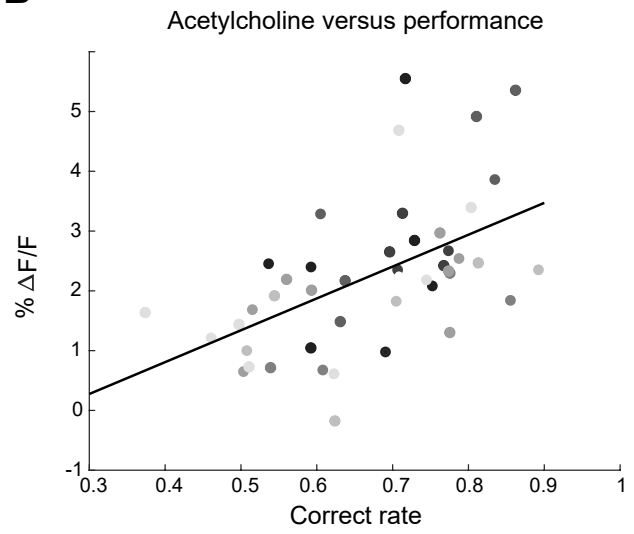

C
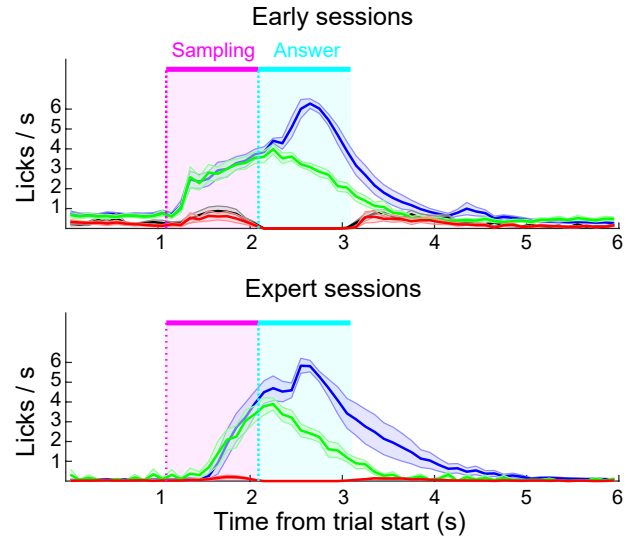

D

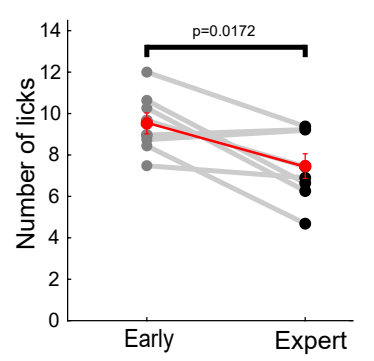

E

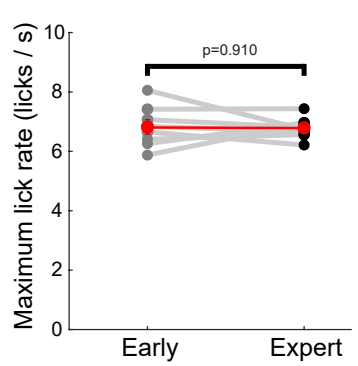

$\mathbf{F}$

G

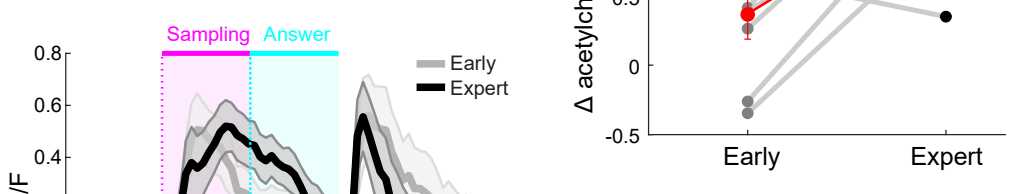

H
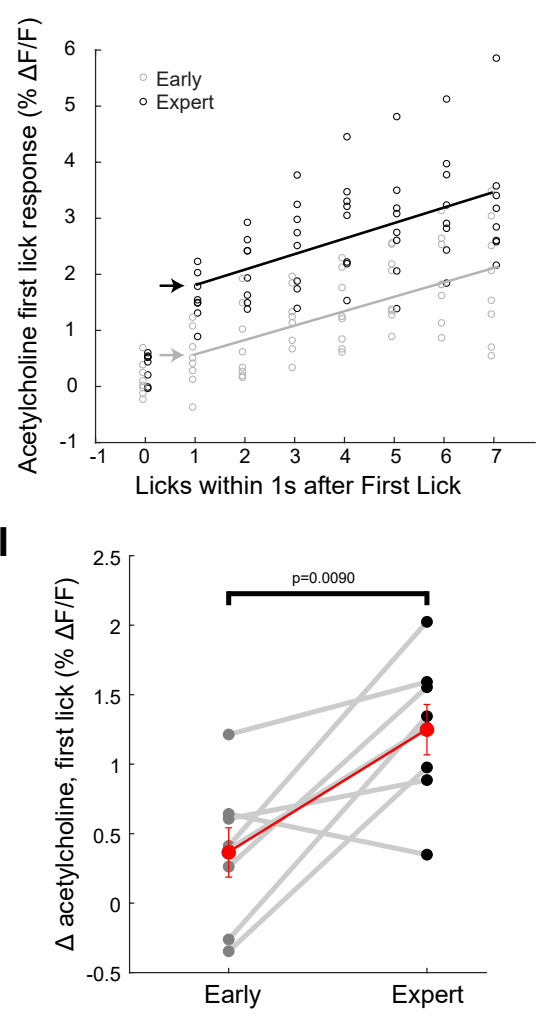

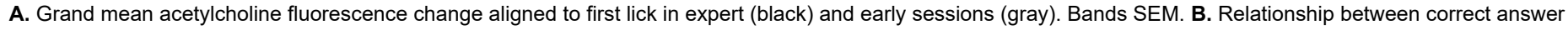

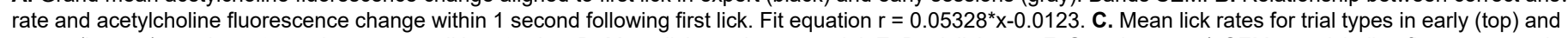

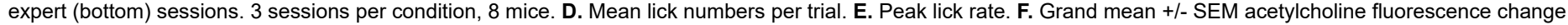

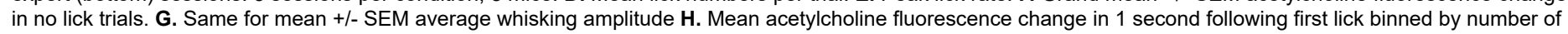
licks in that period. Black, 3 expert sessions / mouse. Gray, 3 early session / mouse. Linear fits from 1-7 licks. I. Difference in acetylcholine fluorescence change between 0 and 1 lick trials for expert (black) and early (gray) sessions. Red, grand mean +/- SEM. 